\title{
Eine kurze Geschichte zum Beginn der medizinischen Radiologie in Deutschland 1896
}

\section{Teil 1 Eine sensationelle Nachricht bricht sich Bahn}

Am 8. November 2020 jährt sich zum 125. Mal der Jahrestag der Entdeckung der Röntgenstrahlen. Wie kaum eine andere Entdeckung haben Röntgens X-Strahlen auch die Medizin stark beeinflusst. Röntgen hatte einen Sonderdruck mit neun Fotografien zu bedeutenden Wissenschaftlern, Kollegen und Freunden versandt.

Zu den Empfängern von Röntgens Postsendung gehörte auch der Berliner Physiker Emil Warburg (1846-1931). Beide kannten sich aus alten Zeiten an der Universität Straßburg. Röntgen wurde als Nachfolger Warburgs auf das Extraordinariat für Physik an der Universität Straßburg berufen, als dieser einen Ruf nach Freigab akzeptierte. Als ordentlicher Professor für Physik an der Berliner Universität war Emil Warburg auch in die Organisation des fünfzigjährigen Gründungsjubiläums der Physikalischen Gesellschaft in Berlin am 4. Januar 1896 involviert. Warburg präsentierte auf der Tagung den einen Tag zuvor erhaltenen Son- derdruck mit den Bildern. Damit wurden die Informationen über die sensationelle Entdeckung erstmals der Öffentlichkeit vorgestellt. Sie befanden sich neben anderen Exponaten an einem weniger auffälligen Ort. Die Bedeutung der Entdeckung wurde daher nur von wenigen Teilnehmern wahrgenommen. Wilhelm von Bezold (1837-1907), Präsident der Gesellschaft für Physik, bedauerte sehr, dass er nichts von den Bildern wusste, sonst hätte er „seine Rede in einem ganz anderen Ton beendet ....".

Einer der führenden Neurologen in Berlin Moritz Jastrowitz (1839-1912) hatte den Nachdruck von Röntgens Röntgenaufnahme der Hand seiner Frau gesehen und verstand sofort die Bedeutung der Entdeckung für die Medizin. Bereits im Januar sprach er vor dem „Verein für innere Medizin“ in Berlin über die neuen Strahlen. In der Deutsche medicinischen Wochenschrift wurden am 30. Januar zwei Vorträge von Jastrowitz abgedruckt, die er am 6. und 20. Januar 1896 über Röntgenversuche mit Kathodenstrahlen und deren diagnostische Verwendbar- keit gehalten hatte. In einem Artikel wurde eine Röntgenaufnahme einer Hand mit einem Glassplitter, der sich in der in der Nähe des Mittelfingergelenks eines vierjährigen Patienten befand. Dieses Röntgenbild war vermutlich am 12. Januar vom Berliner Physiker Paul Spies (1862-1932) von der Urania in Berlin aufgenommen worden.

In dem DMW-Bericht hieß es: „Dieser Aspekt ist offensichtlich für die Medizin wichtig. Die Chirurgie könnte ihn sich zunutze machen, um Knochenbilder einer lebenden Person zu erstellen. Frakturen, Verrenkungen, Aufblähungen und Fremdkörper werden gut unterscheidbar sein; ich weise Sie auf die scharfen Konturen der Fingergelenke hin, die auf dem Foto hell erscheinen; wir werden in die Gelenke hineinschauen können. Es ist auch möglich, dass wir in das Innere des Körpers schauen können, in die Bauchhöhlen, wenn die Strahlung die Wände passiert, und einige Veränderungen erkennen, vielleicht dichtere Tumore, die für Röntgenstrahlen weniger durchlässig sind“. 
Ius den Sitzungsberichten der Nürburger Plissik.medic, Gesellsehaft $189 \%$

\section{W. C. Röntgen: Veher eine neut Art von Strahlen.}

(Vorläufige Mittheilung.)

1. Lässt man durch eine Hittorf'sche Vacuumröhre, oder einen genügend evacuirten Lenard'sehen, Crookes'schen oder ähnliehen A pparat die Entladungen eines grösseren Ruhmkor:lf's gehen und bedeckt die Röhre mit einem ziemlich eng anliegenden Mantel aus diinsem, schwarzem Carton, so sieht man in dem vollstïndig verdunkelten Zimmer einen in die Nähe des Apparates gebrachten, mit Bariumplatincyanür angestrichenen Papiersehirm bei jeder Entladung hell aufleuchten, fluoresciren, gleichgültig ob die angestrichene oder die andere Seite des Schirmes dem Entladungsapparat zugewendet ist. Die Fluorescenz ist noch in $2 \mathrm{~m}$ Entfernung vom Apparat bemerkbar.

Man ïberzeugt sich leicht, dass die Ursache der Flnorescenz vom Entladungsapparat und von keiner anderen stelle der Leitung ausgeht.

2. Das an dieser Erseheinung zunächst Auffallende ist, dass durch die schwarze Cartonhïlse, welche keine sichtbaren oder ultravioletten Strahlen des Sonnen- oder des elektrischen Bogenlichtes durchlïsst, ein Agens hindurehgeht, das im Stande ist, lebhafte Fluorescenz zu erzeugen, und man wird deshalb wohl zuerst untersuchen, ob auch andere Körper diese Eigenschaft besitzen.

Man findet bald, dass alle Körper für dasselbe dureblässig sind, aber in sehr versehiedenem Grade. Einige Beispiele führe ich an. Papier ist sehr durehlässig: ${ }_{1}$ ) linter einem eingebun. denen Buch von ea. 1000 Seiten sah ich den Fluorescenzsehirm noch deutlich leuchten; die Druckerschwärze bietet kein merkliches Hinderniss. Ebenso zeigte sich Fluoreseenz hinter einem doppelten Whistspiel; eine einzelne Karte zwischen Apparat

1) Mit „Durchlässigkeit" eines Körpers bezeichne ich das Verhältniss der Helligkeit eines dicht hinter dem Körper gehaitenen Fluorescenzsehirmes zu der. jenigen Helligkeit des Schirmes, weleher dieser unter denselben Verhältnissen aber ohne \%wisehensehaitung des Körpers zeigt.

Erste Seite des Sonderdrucks „Über eine neue Art von Strahlen“ 1896 (Bildquelle @ Archiv Deutsches Röntgen-Museum).

Am 5. Januar 1896 berichtete die Wiener Tageszeitung „Die Presse“ über die sensationelle Entdeckung eines Physikers aus Würzburg. Bereits am 7. und 8. Januar veröffentlichte die Frankfurter Zeitung in ihrem Feuilleton zwei ausführliche Berichte über die Entdeckung der Röntgenstrahlen und die neuen Möglichkeiten, lebende Knochen zu sehen. Die neuen Möglichkeiten in der Medizin wurden vorausschauend disku- tiert. Hier war zu lesen: „Eine sensationelle Entdeckung. In den Wiener Gelehrtenkreisen erregt derzeit die Nachricht einer Entdeckung von Wilhelm Conrad Röntgen, Professor für Physik an der Universität Würzburg, Aufsehen. Wenn sich diese bewahrheitet, dann handelt es sich um ein epochales Ergebnis exakter Forschung auf seine Weise, das sowohl im physikalischen als auch im medizinischen Bereich ganz seltsame Folgen haben könnte. ... Am überraschendsten ist das Bild einer menschlichen Hand, das durch die erwähnte Fotografie entsteht, um deren Finger die Ringe frei zu schweben scheinen. Die Weichteile der Hand sind nicht sichtbar. ..."

\section{Die Aufmerksamkeit des Kaisers}

Diese Nachrichten erreichten auch den deutschen Kaiserhof. Begeistert von neuen Technologien lud Kaiser Wilhelm II, der sich gerne in der Rolle des „Förderers von Wissenschaft und Technik“ präsentierte, Röntgen ein, seine Entdeckung vorzustellen. Die Präsentation fand am Sonntag, 12. Januar, um fünf Uhr nachmittags im Sternensaal des Berliner Schlosses statt. Der bei der Vorführung ebenfalls anwesende Generalstab diskutierte später beim Abendessen mit Roentgen neue Möglichkeiten, Materialfehler in Geschützen und Gewehren mit Hilfe von Röntgenstrahlen zu prüfen. Roentgen versprach, sich um das Problem zu kümmern. Die Antwort zu diesen Fragen erfolgte im Frühjahr 1897. Röntgen übersandte mit erläuternden Erklärungen das Röntgenbild eines seiner Jagdgewehre an den Kaiser.

Wenige Tage später nach der Audienz am Kaiserhof wurde Röntgen vom Präsidenten des Deutschen Reichstags Rudolf Freiherr von Buol-Berenberg (1842-1902) eingeladen, seine Experimente im Reichstag und im Bundesrat in Berlin vorzustellen. Röntgen lehnt diese Einladung jedoch ab. Am 30. Januar übernahm Paul Spies die Präsentation.

\section{Verwendete und weiterführende Literatur}

Schreiner H, Geschichte der Physikalischen Gesellschaft zu Berlin. First published: Januar 1995, S. F47. https://doi.org/10.1002/phbl Jastrowitz M, Die Roentgen'schen Experimente mit Kathodenstrahlen und ihre diagnostische Verwertung. Vorgetragen im Verein f. innere Medicin am 6. und 20. Januar 1896 (pp. 65-67, 2 Abb.), DMW, 22/ 5.-Leipzig, Georg Thieme Verlag, 30. Januar 1896, 4, pp. 65-96 


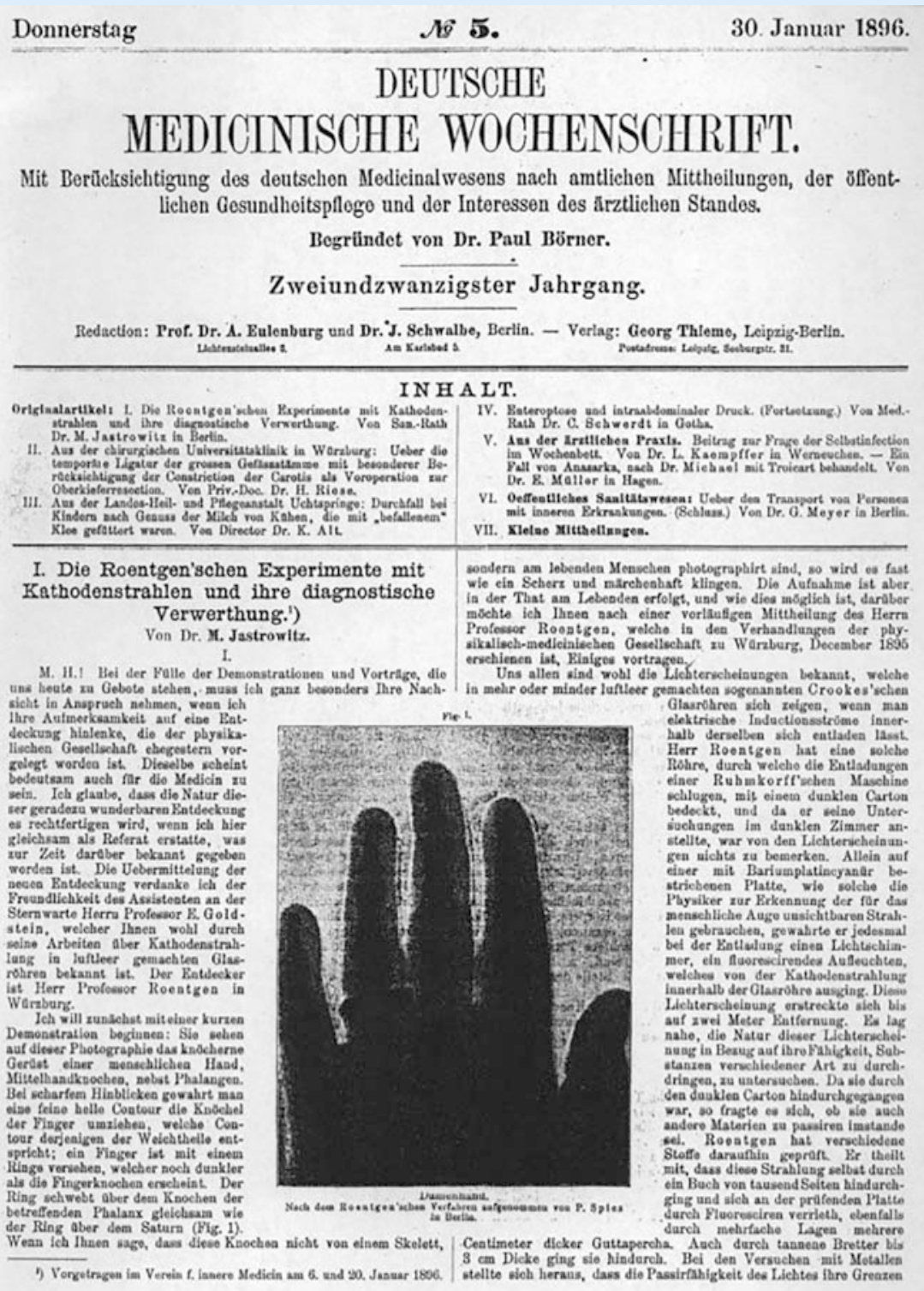

Erste Seite der Publikation der Vorträge von Moritz Jastrovitz in der DMW 1896 (Bildquelle @ Archiv Deutsches Röntgen-Museum).

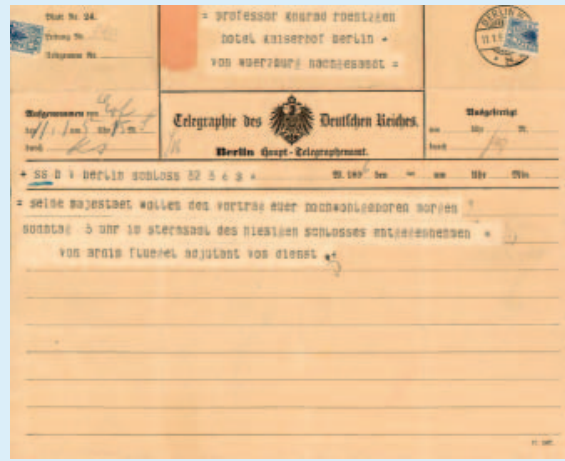

Telegramm des Adjutaten von Kaiser Wilhelm II mit der Einladung zum Vortrag im Berliner Schloss (Bildquelle @ Archiv Deutsches Röntgen-Museum).

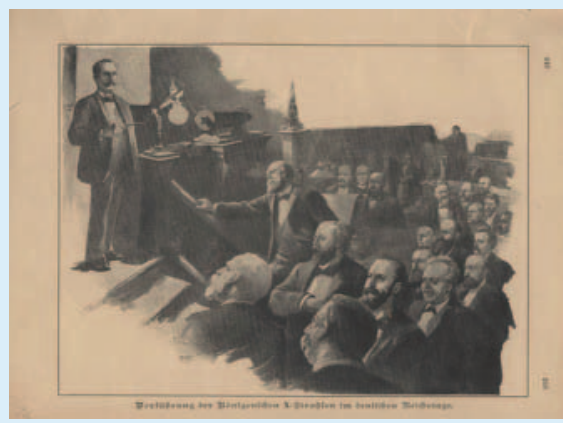

Vortrag über Röntgenstrahlen von Paul Spies im Deutschen Reichstag (Bildquelle (c) Archiv Deutsches Röntgen-Museum).

Spies P, Über Röntgensche Strahlen. Populärer Experimentalvortrag, geh. In der Urania zu Berlin. Berlin: Paetel 1896, 8, (popuäre Schriften, Hrsg. V. d. Urania, 39)

Feuilleton der Frankfurter Zeitung 40, Nr. 7 (Dienstag, den 7. Januar 1896), Zweites Morgenblatt, Feuilleton; 40, Nr. 8 (Mittwoch, den 8. Januar 1896), Abendblatt, Kleines Feuilleton. 